\title{
CD24 promotes the proliferation and inhibits the apoptosis of cervical cancer cells in vitro
}

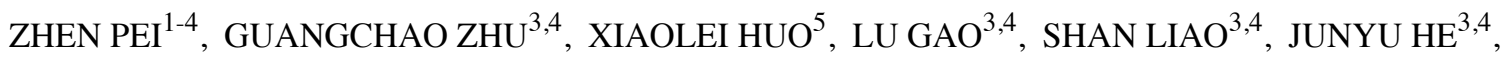

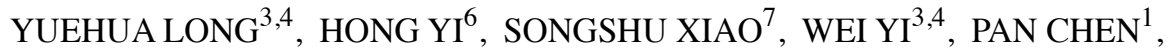 \\ XIAOLING LI ${ }^{1,3,4}$, GUIYUAN LI ${ }^{1,3,4}$ and YANHONG ZHOU ${ }^{1,3,4}$ \\ ${ }^{1}$ Hunan Provincial Tumor Hospital and The Affiliated Tumor Hospital of Xiangya School of Medicine, \\ Central South University, Changsha, Hunan 410006; ${ }^{2}$ Department of Physiology, Changzhi Medical College, \\ Changzhi, Shanxi 046000; ${ }^{3}$ Cancer Research Institute, Central South University; ${ }^{4}$ Key Laboratory of Carcinogenesis \\ of the Chinese Ministry of Health and Key Laboratory of Carcinogenesis and Cancer Invasion of the \\ Chinese Ministry of Education, Cancer Research Institute, Central South University, Changsha, Hunan 410078; \\ ${ }^{5}$ Department of Histology and Embryology, Changzhi Medical College, Changzhi, Shanxi 046000; \\ ${ }^{6}$ Research Center of Carcinogenesis and Targeted Therapy, Xiangya Hospital, Central South University, \\ Changsha, Hunan 410008; ${ }^{7}$ Department of Gynecology and Obstetrics, The Third Xiangya Hospital, \\ Central South University, Changsha, Hunan 410013, P.R. China
}

Received August 31, 2015; Accepted October 22, 2015

DOI: $10.3892 /$ or.2015.4521

\begin{abstract}
The protein CD24 is a cell surface protein that appears to function as an adhesion molecule; its expression has been shown to correlate with prognosis in a variety of tumors. Herein, we investigated the possible role and mechanism of CD24 in cervical cancer. Our results showed that CD24 was overexpressed in cervical cancer tissues compared with that in the adjacent non-cancerous tissues by qPCR, immunohistochemistry and western blotting technologies. To explore the possible mechanism of CD24 in cervical cancer, we elucidated the effect of CD24 on the proliferation and apoptosis of cervical cancer HeLa cells and found that a considerable increase in cell proliferation was observed in the HeLa cells with CD24 overexpession. The rate of cell apoptosis was decreased in the HeLa/CD24 cells compared with the HeLa or HeLa/vector cells. Cell apoptosis is closely related with a reduction in mitochondrial membrane potential $(\Delta \Psi \mathrm{m})$ and an increase in intracellular reactive oxygen species (ROS)
\end{abstract}

Correspondence to: Dr Yanhong Zhou or Professor Guiyuan Li, Cancer Research Institute, Central South University, 110 Xiangya Road, Changsha, Hunan 410078, P.R. China

E-mail: zhouyanhong@csu.edu.cn

E-mail: lgy@csu.edu.cn

Abbreviations: CD24, CD24 molecule; HPV, human papillomavirus; MAPK, mitogen-activated protein kinase; ROS, reactive oxygen species; FBS, fetal bovine serum; PI, propidium iodide; CFE, colony formation efficiency; DCFH-DA, 2',7'-dichlorofluorescein diacetate; IHC, immunohistochemistry; GADPH, glyceraldehyde-3phosphate dehydrogenase

Key words: CD24, cervical cancer, apoptosis, proliferation and calcium ion $\left(\mathrm{Ca}^{2+}\right)$ concentrations. Our results showed that overexpression of CD24 in the cervical cancer HeLa cells, led to an increase in $\Delta \Psi \mathrm{m}$ and a decrease in intracellular ROS and $\mathrm{Ca}^{2+}$ concentrations. Furthermore, we found that CD24 was correlated with dysregulation of the MAPK signaling pathway in cervical cancer tissues in vitro. At the same time, we found that CD24 overexpression affected the expression of p38, JNK2 and c-Jun in vitro. In summary, our results suggest that CD24 is upregulated in cervical cancer tissues and plays its functions by affecting the MAPK signaling pathway in cervical cancer.

\section{Introduction}

Cervical cancer is the second most prevalent cancer in females worldwide (1). Human papillomavirus (HPV) infection is a sexually transmitted infection and is a risk for cervical cancer. However, in addition to HPV infection, other factors exist that influence the risk of developing cervical cancer $(2,3)$. Dysregulated activation of many genes, such as CD44, CD24, CD38, FRA-1 and SOX9 has been implicated in cervical cancer (4-8). miRNAs are closely related to the occurrence and regulation of cervical cancer (9). However, the etiology of cervical carcinoma remains poorly understood.

Cluster of differentiation (CD) 24 was originally described as a B lymphocyte marker. CD24 is a heavily glycosylated cell surface protein that appears to be associated with aggressive cancers involving invasion and metastasis $(10,11)$. Huang and Lee found that CD24 overexpression is a predictor of decreased long-term survival in patients with cervical carcinoma and that CD24 expression is a potential prognostic biomarker for cervical carcinomas (12). Sung et al found that CD24 expression is an independent prognostic marker in patients with cervical squamous cell carcinoma, even following 
adjuvant treatment after surgery. The results showed that new therapeutic strategies targeting CD24 expression stratified by subgroups may have important clinical implications (13). Kwon et al suggested that CD24 expression is a significant independent prognostic factor for distant metastasis-free survival in patients with uterine cervical squamous cell carcinoma (14). Although some evidence was reported, the significant roles of CD24 in cervical cancer development are still elusive.

In the present study, we examined the expression levels of CD24 in cervical cancer tissues. At the same time, we studied the influence of CD24 on the cell proliferation and apoptosis in a cervical cancer cell line and explored the possible mechanisms.

\section{Materials and methods}

Cell culture. One identified general human cervical cancer cell line, HeLa, was cultured in RPMI-1640 (HyClone, Logan, UT, USA) supplemented with $10 \%$ fetal bovine serum (FBS) (Gibco by Life Technologies ${ }^{\mathrm{TM}}$, Grand Island, NY, USA), $100 \mathrm{U} / \mathrm{ml}$ penicillin and $100 \mu \mathrm{g} / \mathrm{ml}$ streptomycin (GE Healthcare Life Sciences, Logan, UT, USA) at $37^{\circ} \mathrm{C}$ in the presence of $5 \% \mathrm{CO}_{2}$.

Patient samples. Sixteen participants were recruited at the Cancer Hospital of Hunan Province, Central South University (Changsha, Hunan, China). Consent forms were obtained from individual patients, and experimental protocols were approved by the Institutional Review Board of the Cancer Hospital of Hunan Province. All subjects enrolled in the study were Chinese. All clinical and biological data were available for the samples (Table I). Cervical cancer tissue and corresponding non-tumor normal tissue were collected, and each biopsy sample was divided into two sections; one was submitted for routine histological diagnosis, and the remaining section was used for qPCR, immunohistochemistry (IHC) and western blotting experiments.

Total RNA extraction and quantitative real-time PCR (qRT-PCR) analysis. Total RNA was extracted from the cervical cancer and corresponding non-tumor normal tissues using TRIzol reagent and cDNA synthesis was carried out using the RevertAid First Strand cDNA Synthesis kit (both from CWBio, Beijing, China) according to the manufacturer's recommendations. qRT-PCR was carried out with GoTaq qPCR Master Mix (Promega, Fitchburg, WI, USA). For detection of CD24 mRNA expression levels, GAPDH was amplified in parallel as an internal control. The sequences of the primers used for qPCR were as follows: CD24 forward, 5'-acccacgcagatttattcca-3' and reverse, 5'-accacgaagagactggetgt-3'; GAPDH forward, 5'-cgaccactttgtcaagctca-3' and reverse, 5'-actgagtgtggcagggactc-3'. The expression of mRNA was assessed by evaluated $\mathrm{CT}$ values. The $\mathrm{CT}$ values were normalized with the expression levels of GAPDH and the relative amount of mRNA specific to each of the target genes was calculated using the $2^{-\Delta \Delta \mathrm{Ct}}$ method (15-19). qPCR was carried out with the Bio-Rad CFK96 $^{\text {TM }}$ Real-Time System (Bio-Rad, Hercules, CA, USA). The data were analyzed by Bio-Rad CFK Manager software (Bio-Rad). Expression of mRNA was assessed by evaluated $\mathrm{CT}$ values and GAPDH was used as an internal control.
IHC and evaluation of staining. IHC was carried out using the peroxidase anti-peroxidase technique following a microwave antigen retrieval procedure. The antibody for $C D 24$ was purchased from Boster Biotechnology (Wuhan, China). The antibody against $C D 24$ (1:100) was overlaid on cervical cancer and corresponding non-tumor normal tissue sections and incubated overnight at $4^{\circ} \mathrm{C}$. Secondary antibody incubation (Santa Cruz Biotechnology, Inc., Santa Cruz, CA, USA) was performed at room temperature for $30 \mathrm{~min}$. Color reaction was developed using a 3,3'-diaminobenzidine tetrachloride (DAB) chromogen solution. All slides were counterstained with hematoxylin. Positive control slides were included in every experiment in addition to the internal positive controls. The specificity of the antibody was determined with matched IgG isotype antibody as a negative control.

Sections were blindly evaluated by two investigators in an effort to provide a consensus on staining patterns by light microscopy (Olympus). CD24 staining was assessed according to the methods described by Hara and Okayasu (20) with minor modifications. Each case was rated according to a score that added a scale of intensity of staining to the area of staining. At least 10 high-power fields were randomly chosen, and $>1,000$ cells were counted for each section. The intensity of staining was graded on the following scale: 0 , no staining; $1+$, mild staining; $2+$, moderate staining; and $3+$, intense staining. The area of staining was evaluated as follows: 0 , no staining of cells in any microscopic fields; $1+,<30 \%$ of the tissue stained positive; $2+$, between 30 and $60 \%$ of the tissue stained positive; $3+,>60 \%$ of the tissue stained positive. The minimum score when summed (extension + intensity) was, therefore, 0 ; and the maximum, 6 . A combined staining score (extension + intensity) of $\leq 2$ was considered to be negative staining (low staining); a score between 3 and 4 was considered to be moderate staining; whereas a score between 5 and 6 was considered to be strong staining. An optimal cut-off level was identified as follows: a staining index score of 0-2 was used to define tumors with negative expression and 3-7 indicated positive expression of these two proteins. Agreement between the two evaluators was 95\%, and all scoring discrepancies were resolved through discussion between the two evaluators.

Construction of the pEGFP-N1-CD24 vector and cell transfection. The coding region of the CD24 gene was generated by PCR with the primer pair: 5'-tattatctcgagatgggcagagcaatggt ggc-3' and 5'-ggcggcgaattcttaagagtagagatgcagaa-3'. PCR was performed under the following conditions: one cycle for $5 \mathrm{~min}$ at $94^{\circ} \mathrm{C}, 30$ cycles for $45 \mathrm{sec}$ at $94^{\circ} \mathrm{C}, 45 \mathrm{sec}$ at $55^{\circ} \mathrm{C}$, and $90 \mathrm{sec}$ at $72^{\circ} \mathrm{C}$, and ended with $10 \mathrm{~min}$ at $72^{\circ} \mathrm{C}$. The fragments were cloned into the TA vector (Promega) and used to transform E. coli JM109 (Takara, Dalian, China). Following selection and propagation, the pure plasmid DNA was prepared by standard methods. The DNA fragments were removed from the TA vector by restriction enzyme digestion with XhoI and EcoR1 (Promega) to subclone into the pEGFP-N1 vector. The fusion sequences were verified by DNA sequencing using ABI 3730. To establish a stable CD24-expressing cell line, the plasmid pEGFP-N1/CD24 or control empty vector pEGFP-N1 was transfected into the HeLa cells, using Lipofectamine (Invitrogen Life Technologies, Carlsbad, CA, USA) according 
Table I. Characteristics of the cervical cancer patients.

\begin{tabular}{ccclc}
\hline Sample & Age, years & HPV type & & Histological diagnosis \\
\hline 1 & 46 & 16 & Cervical intermediately differentiated squamous cell cancer & Stage \\
2 & 60 & 16 & Cervical intermediately differentiated squamous cell cancer & IIa2 \\
3 & 60 & $16,53,58$ & Cervical poorly differentiated squamous cell cancer & IIa2 \\
4 & 47 & 16 & Cervical intermediately differentiated squamous cell cancer \\
5 & 49 & 18 & Cervical intermediately differentiated squamous cell cancer & IIb \\
6 & 49 & 6 & Cervical highly differentiated squamous cell cancer & IIa1 \\
7 & 43 & 16 & Cervical intermediately differentiated squamous cell cancer \\
8 & 48 & 16 & Cervical intermediately differentiated squamous cell cancer \\
9 & 40 & 16, CP8304 & Cervical intermediately differentiated squamous cell cancer \\
10 & 46 & 16 & Cervical intermediately differentiated squamous cell cancer \\
11 & 39 & 16 & Cervical intermediately differentiated squamous cell cancer \\
12 & 56 & $(-)$ & Cervical poorly differentiated squamous cell cancer & Ib2 \\
13 & 50 & 16 & Cervical poorly differentiated squamous cell cancer \\
14 & 46 & 16 & Cervical intermediately differentiated squamous cell cancer \\
15 & 36 & 16,58 & Cervical poorly differentiated squamous cell cancer \\
16 & 60 & 59 & Cervical intermediately differentiated squamous cell cancer \\
\hline
\end{tabular}

${ }^{a}$ The International Federation of Gynecologists and Obstetricians (FIGO) stage: 2009. HPV, human papillomavirus.

to the manufacturers' instructions, followed by G418 selection. The stable transfectants, HeLa/CD24 and HeLa/vector, were isolated and the transcription of CD24 protein was determined by qPCR and western blot experiments.

In vitro $C C K-8$ assay for cellular viability. Cell viability was measured with Cell Counting Kit-8 (CCK-8) assay (7Sea Pharmatech Co., Ltd., Shanghai, China). Cells were prepared in 96-well cell culture plates at a cellular density of $5 \times 10^{3}$ cells/well for the HeLa/CD24, HeLa/vector and HeLa cells at $37^{\circ} \mathrm{C}$ for $24 \mathrm{~h}$. The cell monolayer was washed three times with phosphate-buffered saline (PBS) containing $1.2 \mathrm{mM} \mathrm{CaCl}_{2}$ and $0.7 \mathrm{mM} \mathrm{MgCl}_{2}$. Then a $1: 10$ diluted CCK-8 solution in RPMI-1640 was added to the cells and incubated for $2 \mathrm{~h}$ at $37^{\circ} \mathrm{C}$. The results were measured by a microplate reader at $450 \mathrm{~nm}$ and are expressed as percentages of the control values. All experiments were conducted in triplicate.

Colony formation assay. Approximately $500 \mathrm{HeLa} / \mathrm{CD} 24$, HeLa/vector and HeLa cells were plated in a 6-well plate with triplicate repeats for each cell group to detect the colony formation efficiency (CFE). When the clone contained $>50$ cells, we washed the plate with PBS and fixed the cells in $4 \%$ paraformaldehyde at room temperature for $10 \mathrm{~min}$. The cells were washed twice and stained with crystal violet for 20 min and then the clone number was counted. The CFE was calculated as: Ratio $=$ (the clone number/the number of planted cell) $\mathrm{x} 100 \%$.

Invasion assay. A total of $2 \times 10^{4} \mathrm{HeLa} / \mathrm{CD} 24, \mathrm{HeLa} /$ vector and HeLa cells were re-suspended in serum-free RPMI-1640 medium and were seeded into a Matrigel (BD Biosciences, Franklin Lakes, NJ, USA.)-coated polycarbonate membrane in the upper chambers of a Transwell apparatus (Corning,
Corning, NY, USA). The lower chambers were loaded with 15\% FBS RPMI-1640 medium. Forty-eight hours later, some of the cells invaded to the lower surface of the upper chambers. Cells on the top surface of the upper chambers were removed by a cotton swab. Cells that invaded to the lower surface were fixed in $10 \%$ formaldehyde, and stained with crystal violet. The cells were observed under a microscope and counted in five different fields (magnification, x100). The assay was repeated in at least three independent experiments.

Effect of CD24 on cervical cancer cell apoptosis. Cell apoptosis was analyzed by flow cytometric analysis using a MoFlo ${ }^{\mathrm{TM}}$ XDP High-Performance Cell Sorter (Beckman Coulter, Brea, CA, USA), propidium iodide (PI) and Hoechst 33342 double staining (Nanjing KeyGen Biotech Co., Ltd., Nanjing, China). Briefly, the HeLa cells (HeLa, HeLa/vector and HeLa/CD24) were seeded at a density of $1 \times 10^{6}$ cells/well into 6 -well culture plates. The cells were collected in an Eppendorf tube at $24 \mathrm{~h}$ and washed twice with PBS by centrifugation. The supernatants were discarded. To detect apoptosis, $500 \mu \mathrm{l}$ PBS, $5 \mu 1$ Hoechst 33342 and $5 \mu 1$ PI were added to each tube, and the contents of the tube were mixed in the dark at room temperature for $15 \mathrm{~min}$, followed by FCM testing (Beckman Coulter). Data were acquired and analyzed using Summit v5.2 software (Beckman Coulter).

Detection of mitochondrial membrane potential by JC-1. The impact of CD24 was measured by flow cytometry using the sensitive and relatively mitochondrion-specific lipophilic cationic probe fluorochrome JC-1. JC-1 accumulates to form J-aggregates and emits red fluorescence in the mitochondria with high membrane potential, yet dissociates into monomers and emits green fluorescence in those that lose crossmembrane electrochemical gradient. The cells were suspended 
Table II. mRNA expression level of CD24 in the cervical cancer and adjacent non-cancerous tissues by qPCR.

\begin{tabular}{llcccccc}
\hline Gene & \multicolumn{1}{c}{ Sample } & $\mathrm{n}$ & $\begin{array}{c}\mathrm{CD} 24 \\
(\mathrm{mean} \pm \mathrm{SD})\end{array}$ & $\begin{array}{c}\text { GAPDH CT } \\
(\text { mean } \pm \mathrm{SD})\end{array}$ & $\begin{array}{c}\Delta \mathrm{CT} \\
(\text { mean } \pm \mathrm{SD})\end{array}$ & $\begin{array}{c}\Delta \Delta \mathrm{CT} \\
(\text { mean } \pm \mathrm{SD})\end{array}$ & Fold $^{\mathrm{a}}$ \\
\hline CD24 & Cervical cancer & 16 & $28.31 \pm 1.05$ & $18.67 \pm 0.64$ & $9.64 \pm 0.43$ & $-1.72 \pm 0.38$ & 3.29 \\
& Non-cancerous tissues & 16 & $29.24 \pm 1.12$ & $17.88 \pm 0.58$ & $11.36 \pm 0.45$ & & $(2.53-4.28)$ \\
\hline
\end{tabular}

${ }^{\mathrm{a}}$ Mean fold-change in expression of the target gene, CD24, relative to the internal control gene, GAPDH, was calculated using the $2^{-\Delta \Delta C t}$ equation previously adopted by Livak and Schmittgen (15): $\Delta \Delta \mathrm{CT}=\left(\mathrm{CT}_{\text {Target }}-\mathrm{CT}_{\mathrm{GAPDH}}\right)$ cervical cancer - $\left(\mathrm{CT}_{\text {Target }}-\mathrm{CT}_{\mathrm{GAPDH}}\right)$ control. At least three replicates of each reaction were performed. CT, threshold cycle; qPCR, quantitative polymerase chain reaction.

in $1 \mathrm{ml}$ of warm staining buffer at $\sim 1 \times 10^{6}$ cells $/ \mathrm{ml}$ and were incubated at $37^{\circ} \mathrm{C}$ for $5 \mathrm{~min}$. Then $1 \mu \mathrm{l}$ of $2 \mathrm{mM} \mathrm{JC}-1(2 \mu \mathrm{M}$ final concentration) was added and the cells were incubated at $37^{\circ} \mathrm{C}$ in $5 \% \mathrm{CO}_{2}$ for $15-30 \mathrm{~min}$. The cells were pelleted by centrifugation, resuspended by gently flicking the tubes, and $500 \mu \mathrm{l}$ PBS was added to each tube. Cells were analyzed with a MoFlo ${ }^{\mathrm{TM}}$ XDP High-Performance cell sorter. Data were acquired and analyzed using Summit v5.2 software.

Intracellular ROS measurement. The production of intracellular reactive oxygen species (ROS) was measured by performing flow cytometry using the oxidation-sensitive probe, 2',7'-dichlorofluorescein diacetate (DCFH-DA) (Applygen, Beijing, China). Briefly, $10 \mathrm{mM}$ DCFH-DA stock solution (in methanol) was diluted 4,000-fold in cell culture medium without serum or other additives to yield a $2.5 \mathrm{mM}$ working solution. After the exposure of human umbilical endothelial cells (HUVECs) to silica nanoparticles for 3 and $24 \mathrm{~h}$, respectively, the cells in 6-well plates were washed twice with PBS and incubated in $2 \mathrm{ml}$ working solution of DCFH-DA at $37^{\circ} \mathrm{C}$ in the dark for $30 \mathrm{~min}$. Then the cells were washed twice with cold PBS and resuspended in the PBS for analysis of intracellular ROS by FACS (Beckman Coulter).

Intracellular $\mathrm{Ca}^{2+}$ concentration assay. Intracellular $\mathrm{Ca}^{2+}$ concentration was measured by means of the fluorescent $\mathrm{Ca}^{2+}$ chelator Fura-2 AM, which permeates into cells where it is cut into Fura-2, resorting within the cells. Fura-2 combines with intracellular $\mathrm{Ca}^{2+}$ to form a fluorescent compound, whose fluorescent intensity is determined at excitation wavelength $340 \mathrm{~nm}$ and emission wavelength $510 \mathrm{~nm}$ in FACS. After treatment, the cells were harvested and rinsed with PBS. The harvested cells were suspended in PBS and incubated with $5 \mu \mathrm{M}$ Fura- $2 \mathrm{AM}$ for $60 \mathrm{~min}$ at $37^{\circ} \mathrm{C}$. During the session of incubation with Fura-2 AM, cell cultures were mildly shaken at intervals of $10 \mathrm{~min}$ aimed to facilitate the combination of Fura- 2 and $\mathrm{Ca}^{2+}$ to form the fluorescent compound. Then, the cells were washed twice and resuspended in PBS for FACS measurement. Data were acquired and analyzed using Summit v5.2 software.

Western blot analysis. The cervical cancer tissues, corresponding non-tumor normal tissues, and HeLa cells were lysed in RIPA buffer (CWBio), and the total protein concentration was determined using Pierce ${ }^{\circledR}$ BCA protein assay kit (Thermo Scientific, Inc., Rockford, IL, USA). Extracts containing
$50 \mu \mathrm{g}$ of proteins were separated on $10 \%$ SDS-PAGE gels and electroblotted onto nitrocellulose membranes (HyClone Laboratories). The membranes were inhibited using tris-buffered saline/Tween-20 (25 mM Tris-HCl, $150 \mathrm{mM}$ $\mathrm{NaCl}$, pH 7.5 and $0.05 \%$ Tween-20) containing 5\% non-fat milk followed by overnight incubation at $4{ }^{\circ} \mathrm{C}$ with the primary antibodies [rabbit anti-human p38 antibody (1:200; catalog no. B7178; Anbo Biotechnology Company, Changzhou, China); rabbit anti-human JNK2 antibody (catalog no. sc-827) and rabbit anti-human c-Jun antibody (1:500; catalog no. sc-1694; Santa Cruz Biotechnology, Inc.); and rabbit anti-Fra-1 antibody (1:300; ImmunoWay Biotechnology Co.)]. Following three washes, the membranes were incubated with a horseradish peroxidase-conjugated secondary antibody (Santa Cruz Biotechnology, Inc.), and the specific signals were visualized using an ECL detection system. Anti-GAPDH antibody (1:3,000; Santa Cruz Biotechnology, Inc.) was used as a loading control.

Statistical analysis. Differences in non-parametric variables were analyzed by the Mann-Whitney U test. Differences in the quantitative variables between groups were analyzed by Student's t-test using SPSS 11.0 program (SPSS, Inc., Chicago, IL, USA). A value of $\mathrm{p}<0.05$ was considered to indicate a statistically significant result.

\section{Results}

CD24 is highly expressed in cervical cancer tissues. To detect the mRNA expression levels of the CD24 molecule in the cervical cancer and adjacent non-cancerous tissues, 16 samples of each were selected to perform qPCR of the CD24 gene. The data were analyzed using the $2^{-\Delta \Delta \mathrm{Ct}}$ method and the fold-change in the expression of these genes relative to the internal control gene, GAPDH, was analyzed. The expression of the CD24 gene was higher in the cervical cancer samples compared with the adjacent non-cancerous tissues, and the normalized CD24 gene expression in cervical cancer was upregulated by 3.29-fold (Table II).

To determine whether the CD24 gene is expressed at a higher level in cervical cancer compared with the adjacent non-cancerous tissues, the protein expression levels of CD24 were further examined by western blotting in 1-4 samples $(\mathrm{p}<0.01$; Fig. 1A). In comparison with the adjacent non-cancerous tissues, the expression level was identified to be greater in the cervical cancer tissues, which corresponded with the qPCR results. 
A a

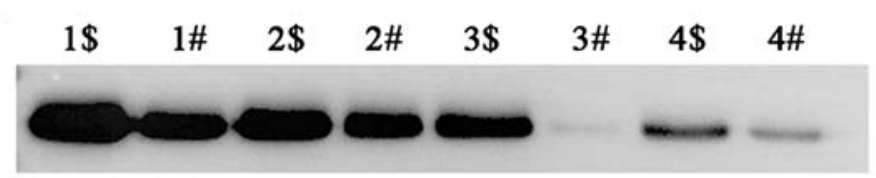

CD24

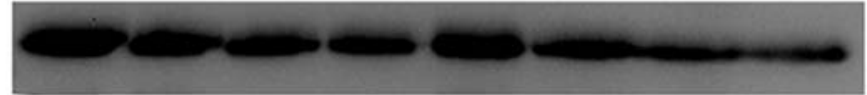

GAPDH

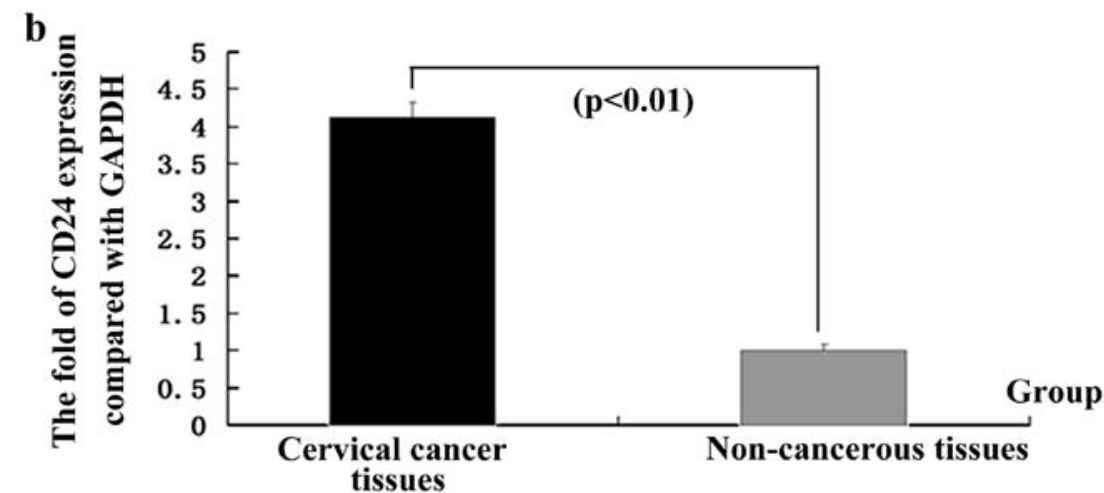

B

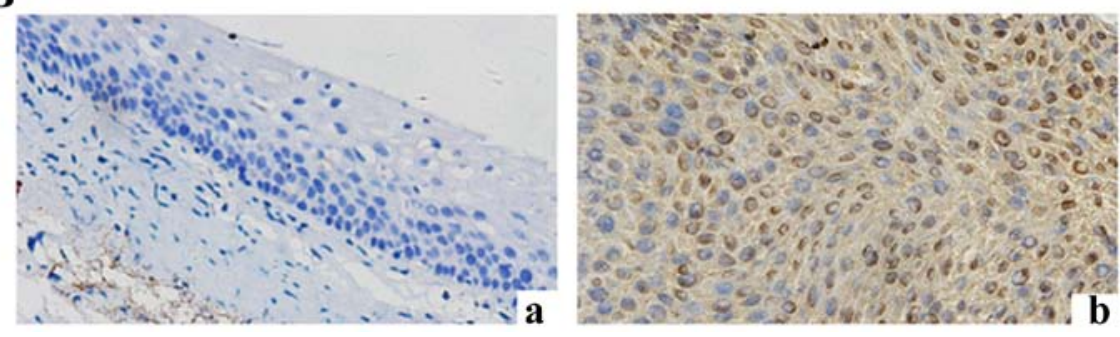

Figure 1. Detection of expression levels of CD24 in the cervical cancer and the adjacent non-cancerous tissues by western blotting and IHC. (A) (a) Western blotting was performed to validate the expression of CD24 in cervical cancer and the adjacent non-cancerous tissues. Cervical cancer and adjacent normal tissues which were used in the detection of mRNA expression levels by qPCR were selected to detect the expression levels of CD24 protein by western blot analysis. $1 \$-4 \$$, cervical cancer tissues; $1 \#-4 \#$, adjacent non-cancerous tissues. Data are representative of three independent experiments. (b) The densitometric analysis of the western blot results. (B) Immunohistochemistry analysis of the expression of CD24 protein in the cervical cancer and the adjacent non-cancerous tissues. Antibody of CD24 protein was used; brown grains denote a positive signal. (a) CD24 staining of cervical epithelial tissue; (b) CD24 staining of cervical cancer tissue. Original magnification, $\mathrm{x} 200$.

Table III. The difference in CD24 expression between the cervical cancer and the adjacent non-cancerous tissues.

\begin{tabular}{lcccccc}
\hline & & \multicolumn{5}{c}{ Score } \\
\cline { 3 - 6 } & $\mathrm{n}$ & $\begin{array}{c}\text { Low (0-2) } \\
\mathrm{n}(\%)\end{array}$ & $\begin{array}{c}\text { Moderate (3-4) } \\
\mathrm{n}(\%)\end{array}$ & $\begin{array}{c}\text { High (5-6) } \\
\mathrm{n}(\%)\end{array}$ & $\chi^{2}$ & P-value \\
\hline Cervical cancer & 16 & $2(12.50)$ & $5(31.25)$ & $9(56.25)$ & 9.26 & $0.0097<0.01$ \\
Non cancerous tissues & 16 & $11(68.75)$ & $4(25.00)$ & $3(18.75)$ & & \\
\hline
\end{tabular}

$\mathrm{p}<0.01$ by Mann-Whitney U test.

To confirm the pattern of CD24 in cervical cancer, IHC was carried out with antibodies against $\mathrm{CD} 24$ protein in the cervical cancer and the adjacent non-cancerous tissues. CD24 was identified as being differentially expressed between the cervical cancer tissues and the adjacent non-cancerous tissues. IHC showed a similar pattern in protein expression with the western blot results. A high score for CD24 was noted in $56.25 \%(9 / 16)$ of the cervical cancer tissues and $18.75 \%$
(3/16) of the adjacent non-cancerous tissues. A low score was found in $12.50 \%(2 / 16)$ and $68.75 \%(11 / 16)$ of the cervical cancer and the adjacent non-cancerous tissues, respectively $(\mathrm{p}=0.0097<0.01)($ Fig. 1B and Table III). This corresponded with the qPCR results.

CD24 promotes the growth and invasion of cervical cancer cells in vitro. To elucidate the function of $\mathrm{CD} 24$ in the growth 

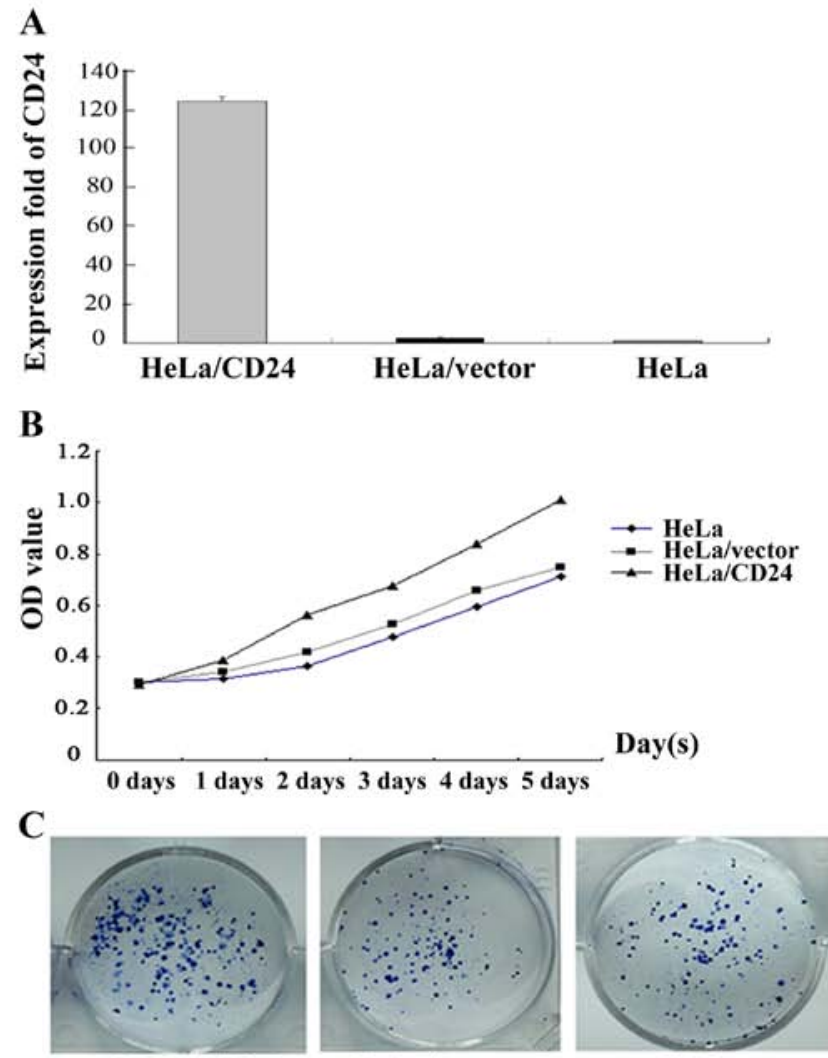

D

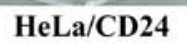

HeLa/vector

HeLa

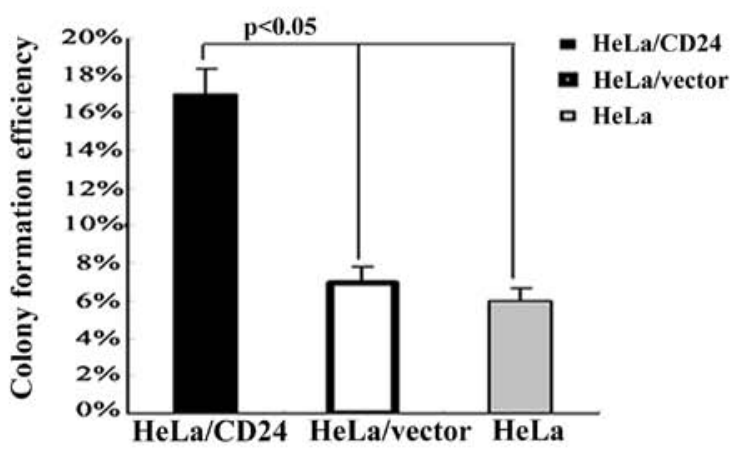

$\mathbf{E}$

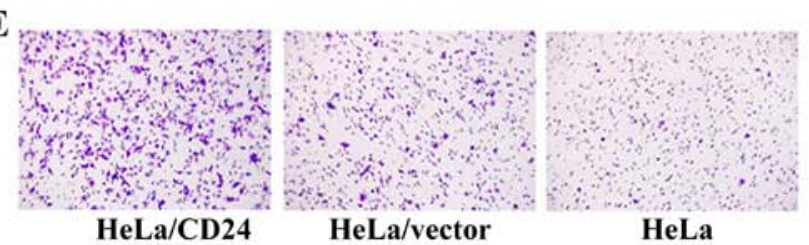

Figure 2. Effect of CD24 on cell proliferation, invasion and apoptosis of cervical cancer cell line, HeLa. (A) CD24 mRNA transcription by RT-PCR. (B) Cell viability of $\mathrm{HeLa}, \mathrm{HeLa} /$ vector and $\mathrm{HeLa} / \mathrm{CD} 24$ cells by CCK-8 assay. (C) Colony formation assay of HeLa, HeLa/vector and HeLa/CD24 cells. (D) Analysis of the colony formation abilities of the HeLa, HeLa/vector and HeLa/CD24 cells. (E) Matrigel invasion analysis of HeLa, HeLa/vector and $\mathrm{HeLa} / \mathrm{CD} 24$ cells. The assay was conducted as three independent experiments. The results are shown as the means \pm standard errors (SE).

of cervical cancer cells, the HeLa cells were transfected with the plasmid pEGFP-N1/CD24 or the control vector to generate CD24-stable expressing HeLa/CD24 or control HeLa/vector cell lines. After demonstrating CD24 mRNA transcription by RT-PCR (Fig. 2A), the spontaneous proliferation of HeLa, $\mathrm{HeLa} /$ vector and $\mathrm{HeLa} / \mathrm{CD} 24$ cells was determined by the

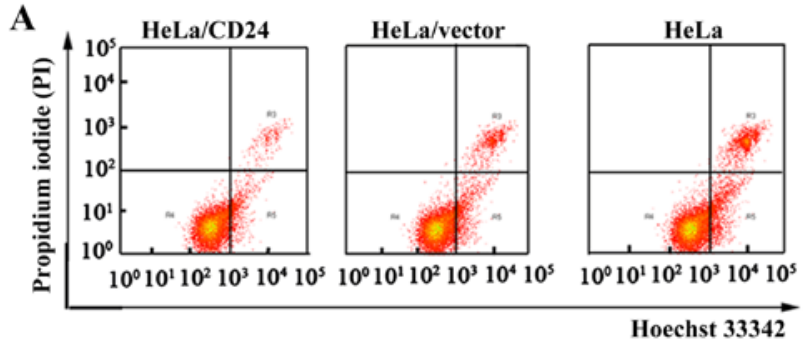

B

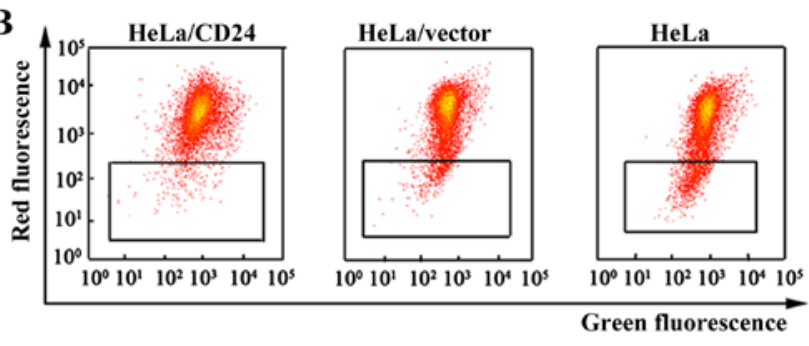

C

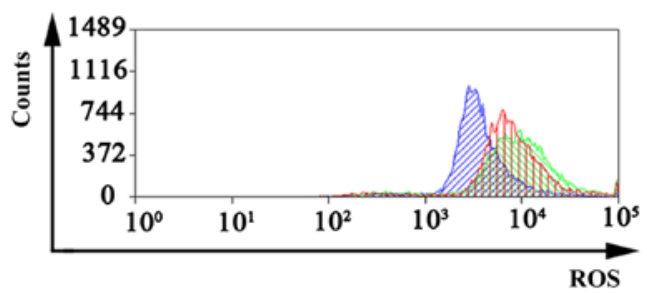

D

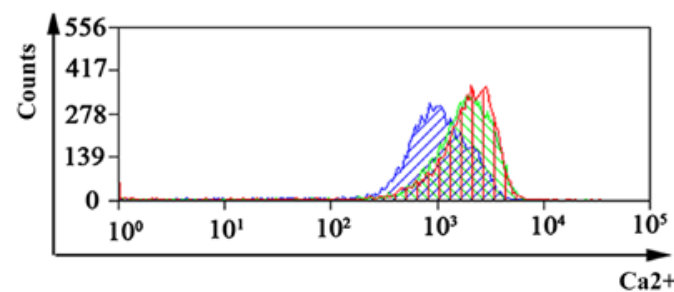

Figure 3. Cell apoptosis, mitochondrial membrane potential $(\Delta \Psi \mathrm{m})$, intracellular reactive oxygen species (ROS) and calcium ion $\left(\mathrm{Ca}^{2+}\right)$ concentrations in the HeLa, HeLa/vector and HeLa/CD24 cells. (A) Cell apoptosis analysis of $\mathrm{HeLa}, \mathrm{HeLa} /$ vector and HeLa/CD24 cells was tested by flow cytometry. The percentage of cell apoptosis for the HeLa/CD24 cells was 3.54, 3.68 and $3.79 \%$ in three independent experiments; the percentage of cell apoptosis for the HeLa/vector cells was 9.24, 9.47 and $9.53 \%$; and the percentage of cell apoptosis for the HeLa cells was 9.89, 10.11 and $10.74 \%$ (p<0.05). (B) $\Delta \Psi \mathrm{m}$ of HeLa, HeLa/vector and HeLa/CD24 cells was tested by flow cytometry. The percent of HeLa/CD24 was $1.58,1.63$ and $1.72 \%$ in three independent experiments, the HeLa/vector was 3.48, 3.56 and $3.74 \%$, the HeLa was 7.89, 7.11 and $7.74 \%(\mathrm{p}<0.05)$. (C) ROS test of HeLa, HeLa/vector and HeLa/CD24 cells was tested by flow cytometry. Blue indicates HeLa/CD24, red indicates $\mathrm{HeLa}$ /vector and green indicates HeLa cells. (D) $\mathrm{Ca}^{2+}$ concentrations test of $\mathrm{HeLa}, \mathrm{HeLa} /$ vector and HeLa/CD24 cells was tested by flow cytometry. Blue indicates $\mathrm{HeLa} / \mathrm{CD} 24$, red indicates HeLa/vector and green indicates HeLa cells. Data are representative of three independent experiments.

CCK-8 assay (Fig. 2B). Clearly, CD24 significantly promoted the proliferation of HeLa cells. Therefore, endogenous CD24 overexpression promoted the proliferation of HeLa cells in vitro.

At the same time, HeLa, HeLa/vector and HeLa/CD24 cells were cultured into a 6-well plate and CFE was observed during 12 days of culture. After the 12 days, $\mathrm{HeLa} / \mathrm{CD} 24$ cells grew well and most clones had reached $>50$ cells, but HeLa and $\mathrm{HeLa} /$ vector cells had fewer cells attached to the plates and formed smaller clones compared to the HeLa/CD24 cells. 
A

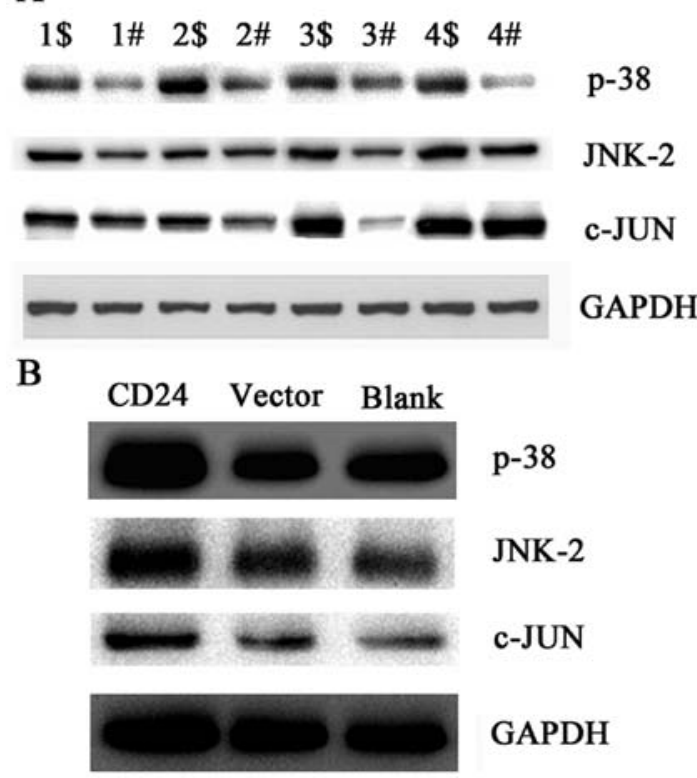

Figure 4. Expression levels of p38, JNK2 and c-Jun protein in cervical cancer tissues and in $\mathrm{HeLa}, \mathrm{HeLa} /$ vector and $\mathrm{HeLa} / \mathrm{CD} 24$ cells. (A) Cervical cancer and normal adjacent tissues used in the detection of CD24 mRNA expression levels by qPCR were selected to detect the expression levels of p38, JNK2 and c-Jun protein by western blot analysis. $1 \$-4 \$$, cervical cancer tissues; 1\#-4\#, adjacent non-cancerous tissues. Data are representative of three independent experiments. (B) Expression levels of p38, JNK2 and c-Jun protein in $\mathrm{HeLa}, \mathrm{HeLa} /$ vector and $\mathrm{HeLa} / \mathrm{CD} 24$ cells. CD24, HeLa cells transfected with pEGFP-N1-CD24. Vector, HeLa cells transfected with pEGFP-N1. Blank, HeLa cells not transfected with the plasmid. Data are representative of three independent experiments.

We counted the number of clones and the statistical analysis showed significant differences in CFE among the HeLa, $\mathrm{HeLa} /$ vector and HeLa/CD24 cells (p<0.01; Fig. 2C and D).

Cell invasion is an important step during tumor metastasis. Thus, we detected the invasion ability of the HeLa, HeLa/vector and HeLa/CD24 cells by Transwell assay in vitro. We found that more $\mathrm{HeLa} / \mathrm{CD} 24$ cells migrated through the Matrigelcoated polycarbonate membrane (Fig. 2E). The results showed that $\mathrm{HeLa} / \mathrm{CD} 24$ cells had higher invasive ability compared to the $\mathrm{HeLa}$ and $\mathrm{HeLa} /$ vector cells.

CD24 inhibits the apoptosis of cervical cancer cells in vitro. We found that CD24 was highly expressed in the cervical cancer tissues by qPCR, western blotting and IHC technologies. To elucidate the function of CD24 in the apoptosis of cervical cancer cells, the apoptosis of HeLa, HeLa/vector and $\mathrm{HeLa} / \mathrm{CD} 24$ cells was tested. We performed a Hoechst 33342/PI double-staining experiment to test the rate of apoptosis in the $\mathrm{HeLa}, \mathrm{HeLa} /$ vector and $\mathrm{HeLa} / \mathrm{CD} 24$ cells. A considerable decrease in the percentage of apoptotic cells was observed for $\mathrm{HeLa} / \mathrm{CD} 24(3.67 \pm 0.28 \%), \mathrm{HeLa} /$ vector $(9.41 \pm 0.83 \%)$ and HeLa cells (10.25 $\pm 0.92 \%)$ (Fig. 3A).

CD24 affects the mitochondrial membrane potential $(\Delta \Psi \mathrm{m})$, $R O S$ and calcium ion $\left(\mathrm{Ca}^{2+}\right)$ concentrations of cervical cancer cells in vitro. Cell apoptosis is closely related with a reduction in $\Delta \Psi \mathrm{m}$ and an increase in intracellular ROS and $\mathrm{Ca}^{2+}$ concentrations. Thus, we tested the influence of CD24 on these three parameters. Our results showed that overexpression of CD24 in cervical cancer HeLa cells, led to an increase in $\Delta \Psi \mathrm{m}$ and inhibition of cell apoptosis (Fig. 3B). ROS results showed that overexpression of CD24 in the HeLa cells, led to a decrease in intracellular ROS (Fig. 3C). $\mathrm{Ca}^{2+}$ experiment results showed that overexpression of CD24 in the HeLa cells, led to a decrease in $\mathrm{Ca}^{2+}$ concentrations and suppressed the apoptosis of cervical cancer cells (Fig. 3D).

CD24 is correlated with dysregulation of the MAPK signaling pathway in cervical cancer tissues. To uncover the possible mechanism of CD24 in cervical cancer, we tested the expression levels of key molecules in the MAPK signaling pathway by western blotting. p38, JNK2 and c-Jun were upregulated in the cervical cancer compared with levels in the adjacent non-cancerous tissues (Fig. 4A). Since CD24 was highly expressed in the cervical cancer tissues, we inferred that CD24 was correlated with dysregulation of the MAPK signaling pathway in cervical cancer tissues in vitro. A positive correlation may exist between the expression of CD24 and the MAPK signaling pathway in cervical cancer.

CD24 overexpression affects the expression of $p 38, J N K 2$ and c-Jun in vitro. To confirm whether CD24 affects the expression of p38, JNK2 and c-Jun in vitro, we detected the expression levels of p38, JNK2 and c-Jun in HeLa, HeLa/vector and $\mathrm{HeLa} / \mathrm{CD} 24$ cells by western blotting. The results showed that $\mathrm{p} 38, \mathrm{JNK} 2$ and c-Jun proteins were highly expressed in the HeLa/CD24 cells compared with levels in the HeLa and $\mathrm{HeLa} /$ vector cells (Fig. 4B). These results suggest that CD24 overexpression affects the expression of p38, JNK2 and c-Jun in vitro.

\section{Discussion}

Cervical cancer is the second most common gynecological malignancy threatening the health of women worldwide and remains a leading cause of cancer-related deaths in women in developing countries (1-3,21-23). One major cause is persistent infection of HPV, leading to abnormal epithelial lesions, with progression to precancerous and invasive cancer $(24,25)$. Dysregulated activation of other genes, such as CD24 and CD44, influences the risk of developing cervical cancer (4-8). Thus, sensitive and specific biomarkers for the early detection of cervical cancer are urgently required to reduce the high morbidity and mortality of this disease.

In the present study, we found that the expression of the CD24 gene was higher in the cervical cancer samples when compared with that in the adjacent non-cancerous tissues and the normalized CD24 gene expression in cervical cancer was upregulated by 3.29 -fold. There was a similar tendency noted by western blot experiments. IHC showed a similar pattern in protein expression with qPCR and western blot results. A high score of CD24 was noted in 56.25\% (9/16) of the cervical cancer tissues and $18.75 \%$ (3/16) of the adjacent non-cancerous tissues. Huang and Lee found that CD24 was overexpressed in invasive cervical carcinoma (12). Kwon et al results showed that positive staining of CD24 expression was found in $58.9 \%$ of the cases (14). Our results corresponded with their results. 
To uncover the potential mechanism of CD24 in cervical cancer, we studied the effect of $\mathrm{CD} 24$ on the proliferation, invasion and apoptosis of cervical cancer HeLa cells. Our results showed that there was a considerable increase in proliferation and invasion of HeLa cells with CD24 overexpession. Compared with the HeLa cells, the rate of apoptotic cells was decreased in the HeLa cells with CD24 overexpession. Overexpression of CD24 in laryngeal squamous cell carcinoma is associated with invasiveness, metastatic potential and high tumor proliferation status (26). Wang et al found that CD24 was associated with enhanced invasiveness of gastric carcinogenesis and a poor prognosis (27). Leelawat et al showed that CD24 expression is linked to the aggressiveness of cholangiocarcinoma cells and the adverse prognosis of cholangiocarcinoma patients (28). CD24 enhanced DNA damage-induced apoptosis by modulating NF- $\mathrm{kB}$ signaling in CD44-expressing breast cancer cells (29). Moreover, CD24 affects the occurrence and development of malignant tumors by cell proliferation, cell invasion and cell apoptosis.

Furthermore, to explore the possible mechanism of CD24 in cervical cancer, we tested the expression levels of key molecules (p38, JNK2 and c-Jun) in the MAPK signaling pathway. Compared with the adjacent non-cancerous tissues, p38, JNK2 and c-Jun were upregulated in the cervical cancer. Thus, a positive correlation between the expression of CD24 and key molecules of the MAPK signaling pathway exist in cervical cancer tissues. At the same time, we studied the effect of the overexpression of CD24 on p38, JNK2 and c-Jun in vitro. The results showed that overexpression of CD24 may upregulate the expression of p38, JNK2 and c-Jun in HeLa cells. MAPKs transduce extracellular signals into a variety of cellular processes, such as cell proliferation, survival, death and differentiation (30). JNK is a family of protein kinases, which are activated by stress stimuli and regulate various cellular processes including proliferation, apoptosis and survival (31). In A549 human non-small cell lung cancer cells, JNK may be specifically required in vivo for the maintenance of the tumor-initiating population of tumor cells rather than for proliferation and survival of the entire cell population (32). Based on the comprehensive literature and our results, we infer that CD24 plays its role by affecting the expression of $\mathrm{p} 38$, JNK2 and c-Jun.

In summary, our results suggest that CD24 promoted the proliferation and inhibited the apoptosis of cervical cancer cells through the MAPK signaling pathway.

\section{Acknowledgements}

The present study was supported by the National Natural Science Foundation of China $(81272975,81172302,81402270$ and 81402307), the Key Project of Hunan Provincial Natural Science Foundation (12JJ2044), the Project of Hunan Provincial Natural Science Foundation (12JJ3121), the Project of Hunan Provincial Development and Reform Commission, the Planned Science and Technology Project of Hunan Province (2010FJ3088, 2012FJ2014 and 2015JC3024), the Planned Project of Department of Health of Hunan Province (B2015-042), and the Hunan Provincial Innovation Foundation for Postgraduats.

\section{References}

1. Parish SL, Swaine JG, Son E and Luken K: Determinants of cervical cancer screening among women with intellectual disabilities: Evidence from medical records. Public Health Rep 128: 519-526, 2013.

2. Duvlis S, Popovska-Jankovic K, Arsova ZS, Memeti S, Popeska Z and Plaseska-Karanfilska D: HPV E6/E7 mRNA versus HPV DNA biomarker in cervical cancer screening of a group of Macedonian women. J Med Virol 87: 1578-1586, 2015.

3. Hu Z, Zhu D, Wang W, Li W, Jia W, Zeng X, Ding W, Yu L, Wang X, Wang L, et al: Genome-wide profiling of HPV integration in cervical cancer identifies clustered genomic hot spots and a potential microhomology-mediated integration mechanism. Nat Genet 47: 158-163, 2015.

4. Xiao S, Zhou Y, Yi W, Luo G, Jiang B, Tian Q, Li Y and Xue M: Fra-1 is downregulated in cervical cancer tissues and promotes cervical cancer cell apoptosis by p53 signaling pathway in vitro. Int J Oncol 46: 1677-1684, 2015.

5. Wobus M, Kuns R, Wolf C, Horn LC, Köhler U, Sheyn I, Werness BA and Sherman LS: CD44 mediates constitutive type I receptor signaling in cervical carcinoma cells. Gynecol 83: 227-234, 2001.

6. Liao S, Xiao S, Zhu G, Zheng D, He J, Pei Z, Li G and Zhou Y: CD38 is highly expressed and affects the PI3K/Akt signaling pathway in cervical cancer. Oncol Rep 32: 2703-2709, 2014.

7. Wu JH, Liang XA, Wu YM, Li FS and Dai YM: Identification of DNA methylation of SOX9 in cervical cancer using methylatedCpG island recovery assay. Oncol Rep 29: 125-132, 2013.

8. Dobo C, Stavale JN, Lima FO, Ribeiro DA, Arias V, Gomes TS and Oshima CT: HSP27 is commonly expressed in cervical intraepithelial lesions of Brazilian women. Asian Pac J Cancer Prev 14: 5007-5010, 2013.

9. Wang J, Wang Q, Liu H, Shao N, Tan B, Zhang G, Wang K, Jia Y, Ma W, Wang N, et al: The association of miR-146a rs2910164 and miR-196a2 rs11614913 polymorphisms with cancer risk: A meta-analysis of 32 studies. Mutagenesis 27: 779-788, 2012.

10. Fujikuni N, Yamamoto H, Tanabe K, Naito Y, Sakamoto N, Tanaka Y, Yanagihara K, Oue N, Yasui W and Ohdan H: Hypoxia-mediated CD24 expression is correlated with gastric cancer aggressiveness by promoting cell migration and invasion. Cancer Sci 105: 1411-1420, 2014.

11. Liu C, Zheng S, Shen H, Xu K, Chen J, Li H, Xu Y, Xu A, Chen B, Kaku H, et al: Clinical significance of CD24 as a predictor of bladder cancer recurrence. Oncol Lett 6: 96-100, 2013.

12. Huang LW and Lee CC: Cluster of differentiation 24 expression is an independent prognostic factor of adverse outcome in cervical carcinoma. Int J Gynecol Cancer 23: 325-330, 2013.

13. Sung CO, Park W, Choi YL, Ahn G, Song SY, Huh SJ, Bae DS, Kim BG and Lee JH: Prognostic significance of CD24 protein expression in patients treated with adjuvant radiotherapy after radical hysterectomy for cervical squamous cell carcinoma. Radiother Oncol 95: 359-364, 2010.

14. Kwon GY, Ha H, Ahn G, Park SY, Huh SJ and Park W: Role of CD24 protein in predicting metastatic potential of uterine cervical squamous cell carcinoma in patients treated with radiotherapy. Int J Radiat Oncol Biol Phys 69: 1150-1156, 2007.

15. Livak KJ and Schmittgen TD: Analysis of relative gene expression data using real-time quantitative PCR and the $2^{-\Delta \Delta C_{\mathrm{T}}}$ method. Methods 25: 402-408, 2001.

16. Zhou Y, Wang W, Zheng D, Peng S, Xiong W, Ma J, Zeng Z, Wu M, Zhou M, Xiang J, et al: Risk of nasopharyngeal carcinoma associated with polymorphic lactotransferrin haplotypes. Med Oncol 29: 1456-1462, 2012.

17. Zheng D, Liao S, Zhu G, Luo G, Xiao S, He J, Pei Z, Li G and Zhou Y: CD38 is a putative functional marker for side population cells in human nasopharyngeal carcinoma cell lines. Mol Carcinog: Jan 28, 2015 (Epub ahead of print). doi: 10.1002/mc.22279.

18. Zhu W, Li J, Su J, Li J, Li J, Deng B, Shi Q, Zhou Y and Chen X: FOS-like antigen 1 is highly expressed in human psoriasis tissues and promotes the growth of $\mathrm{HaCaT}$ cells in vitro. Mol Med Rep 10: 2489-2494, 2014.

19. Zheng D, Zhu G, Liao S, Yi W, Luo G, He J, Pei Z, Li G and Zhou Y: Dysregulation of the PI3K/Akt signaling pathway affects cell cycle and apoptosis of side population cells in nasopharyngeal carcinoma. Oncol Lett 10: 182-188, 2015.

20. Hara A and Okayasu I: Cyclooxygenase-2 and inducible nitric oxide synthase expression in human astrocytic gliomas: Correlation with angiogenesis and prognostic significance. Acta Neuropathol 108: 43-48, 2004. 
21. Sun Y, Zhang R, Zhou S and Ji Y: Overexpression of Notch1 is associated with the progression of cervical cancer. Oncol Lett 9: 2750-2756, 2015

22. Han J and Guo J: Current evidence and potential mechanisms of therapeutic action of PEDF in cervical cancer treatment. Curr Mol Med 15: 446-455, 2015.

23. Ali SF, Ayub S, Manzoor NF, Azim S, Afif M, Akhtar N, Jafery WA, Tahir I, Farid-Ul-Hasnian $S$ and Uddin N: Knowledge and awareness about cervical cancer and its prevention amongst interns and nursing staff in Tertiary Care Hospitals in Karachi, Pakistan. PLoS One 5: e11059, 2010.

24. Swangvaree SS, Kongkaew P and Ngamkham J: Frequency and type-distribution of human papillomavirus from paraffinembedded blocks of high grade cervical intraepithelial neoplasia lesions in Thailand. Asian Pac J Cancer Prev 14: 1023-1026, 2013.

25. Origoni M, Carminati G, Rolla S, Clementi M, Sideri M, Sandri MT and Candiani M: Human papillomavirus viral load expressed as relative light units (RLU) correlates with the presence and grade of preneoplastic lesions of the uterine cervix in atypical squamous cells of undetermined significance (ASCUS) cytology. Eur J Clin Microbiol Infect Dis 31: 2401-2406, 2012.

26. Shi Y, Gong HL, Zhou L, Tian J and Wang Y: CD24: A novel cancer biomarker in laryngeal squamous cell carcinoma. ORL J Otorhinolaryngol Relat Spec 74: 78-85, 2012.
27. Wang YC, Wang JL, Kong X, Sun TT, Chen HY, Hong J and Fang JY: CD24 mediates gastric carcinogenesis and promotes gastric cancer progression via STAT3 activation. Apoptosis 19: 643-656, 2014.

28. Leelawat K, Keeratichamroen S, Leelawat S and Tohtong R: CD24 induces the invasion of cholangiocarcinoma cells by upregulating CXCR4 and increasing the phosphorylation of ERK1/2. Oncol Lett 6: 1439-1446, 2013.

29. Ju JH, Jang K, Lee KM, Kim M, Kim J, Yi JY, Noh DY and Shin I: CD24 enhances DNA damage-induced apoptosis by modulating NF- $\kappa \mathrm{B}$ signaling in CD44-expressing breast cancer cells. Carcinogenesis 32: 1474-1483, 2011.

30. Huang P, Han J and Hui L: MAPK signaling in inflammationassociated cancer development. Protein Cell 1: 218-226, 2010.

31. You H, Lei $P$ and Andreadis ST: JNK is a novel regulator of intercellular adhesion. Tissue Barriers 1: e26845, 2013.

32. Okada M, Shibuya K, Sato A, Seino S, Watanabe E, Suzuki S, Seino $M$ and Kitanaka C: Specific role of JNK in the maintenance of the tumor-initiating capacity of A549 human non-small cell lung cancer cells. Oncol Rep 30: 1957-1964, 2013. 